\title{
Coexistence Strategies and Capacity Theorems of Interference Awareness Cognitive Radio
}

\author{
Na Yi, Yi Ma and Rahim Tafazolli \\ Centre for Communication Systems Research \\ The University of Surrey, Guildford, UK \\ e-mails: \{n.yi, y.ma, r.tafazolli\}@ surrey.ac.uk
}

\begin{abstract}
Interference awareness cognitive radio (IACR) is a special paradigm of cognitive radios, which utilizes spatial spectrum holes for frequency reuse. The primary contributions of this work are in two folds: 1) find the fundamental capacity limit of IACR with perfect channel side information; 2) find the achievable rate for the scenario, where the channel gain between the secondary transmitter and the primary receiver is not known, but the secondary transmitter has the localization information about all network nodes. Numerical results show the relationship between the channel capacity, rate penalty, outage probability, and channel gains. It is shown that the localization information can help the IACR system, but cannot offer comparable performance with the case with perfect channel side information.
\end{abstract}

\section{INTRODUCTION}

Federal Communications Commission (FCC) frequency allocation table revealed that the number of wireless services has grown almost exponentially over the last twenty years [1]. Spectrum allocation bodies such us FCC in US and Ofcom in UK warned that the rapid development of wireless systems has resulted in severe shortage of the primary radio spectrum. Meanwhile, traditional fixed spectrum management resulted in inefficient utilization of the radio spectrum [2]. All of these motivated a dynamic and flexible framework for spectrum and radio resource management referred to as cognitive radio.

The original idea of cognitive radio presented in the article [3] conveys a general framework, whereby a wireless system or device utilizes any available network side information such as channel conditions, codebooks, and even messages communicated between existing wireless devices to determine the efficient strategy of spectrum sharing. Utilizing advanced signal processing and dynamic spectrum allocation policies, cognitive radio techniques support new wireless users operating in the allocated spectrum, without degrading performance of existing primary users.

In the last decade, the majority of research effort was paid for spectrum sensing cognitive radio (SSCR), which is one of special paradigm of the general cognitive radio. The idea came about the fact evident by FCC [4] that there exist temporary spectrum holes in both the licensed and unlicensed frequency bands. Cognitive receivers periodically sense the radio spectrum, and perform opportunistic frequency reuse over the spectrum holes to improve the spectral efficiency. Recently, an overlay cognitive radio technique has been presented in the literature [5]. Cognitive transmitters intelligently utilize

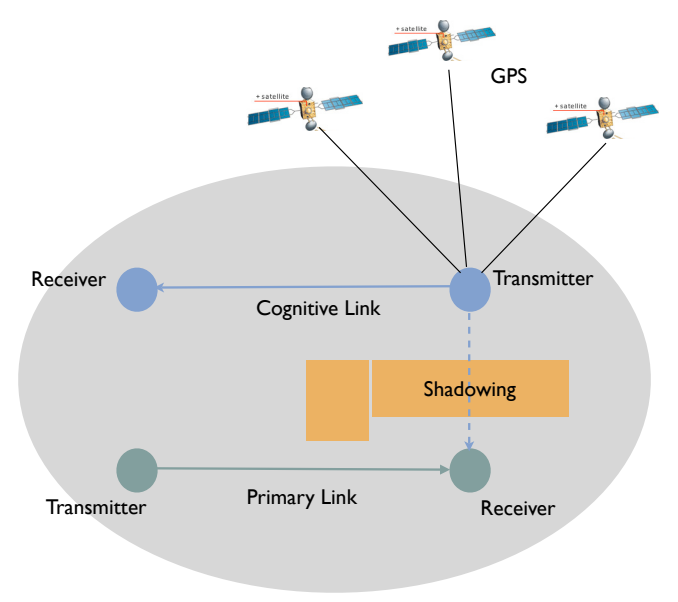

Fig. 1: An example of IACR networks.

all available network side information to perform optimum frequency reuse over the entire frequency band. However, there are many problems remain unsolved in the area of information theory.

The primary focus of this paper is about another paradigm of cognitive radios referred to as interference-awareness cognitive radio (IACR). Fig. 1 depicts an example of the smallest IACR networks accommodating one primary link and one cognitive link which is often referred to as secondary link in literatures. An extreme case for the secondary link to reuse the frequency band occupied by the primary link is that the channel gain between the secondary transmitter and the primary receiver is null. However, there is no such an extreme case in practice. In the IACR paradigm, cognitive users utilize channel side information to evaluate the interference potentially caused by the cognitive transmitter to primary receivers. Communication between cognitive users occurs only if the interference power is below an acceptable threshold. This case is defined as the spatial spectrum hole that offers the opportunity for frequency reuse. The problems this work seeks to address are mainly in two folds:

1) With the availability of channel side information, what is the channel capacity of the IACR paradigm in the Guassian channel? Moreover, what is the optimum power setup at the secondary transmitter? These problems will 
receive a careful investigation in Section III.

2) The process of estimating channel between the secondary transmitter and the primary receiver interferes with the primary communication. This motivates us to utilize localization information to determine the spatial spectrum hole for frequency reuse. This technique will be addressed in Section IV in terms of outage probability and efficient power control.

Remark: The technical contents of this paper are based on the smallest cognitive radio network as depicted in Fig. 1 so that our primary focus is on the fundamental issue in the area of network information theory.

\section{Mathematical Description of IACR}

Consider the situation where the primary transmitter sends information $\mathrm{x}_{1}$ with the power $P_{1}$ to its corresponding receiver via the communication channel $\mathrm{a}_{11}$. The received information at the primary receiver is expressible as

$$
\overline{\mathrm{y}}_{1}=\mathrm{a}_{11} \mathrm{x}_{1}+\mathrm{v}_{1}
$$

where $\mathrm{v}$ is the additive white Gaussian noise with zero mean and variance $\mathcal{N}_{0}$. The achievable rate of this communication (denoted by $R_{11}$ ) is upper bounded by the Shannon capacity, i.e., $R_{1}<\mathcal{C}\left[\left(P_{1} \mathrm{a}_{11}^{2}\right) /\left(\mathcal{N}_{o}\right)\right]$, where $\mathcal{C}[x]=\frac{1}{2} \log _{2}(1+x)$.

Meanwhile, the secondary transmitter wants to reuse the same frequency band to send the information $\mathrm{x}_{2}$ with the power $P_{2}$. The secondary communication occurs under the following condition

$$
P_{2} \mathrm{a}_{21}^{2}<\eta
$$

where $a_{21}$ is the channel gain between the secondary transmitter and the primary receiver, and $\eta$ the power threshold. In the situation of coexistence, the received information at the primary receiver (1) is replaced by

$$
\mathrm{y}_{1}=\mathrm{a}_{11} \mathrm{x}_{1}+\mathrm{a}_{21} \mathrm{x}_{2}+\mathrm{v}_{1}
$$

The threshold $\eta$ is carefully chosen so as not to influence considerably the capacity of the primary communication, i.e.,

$$
\Delta \mathcal{C}=\mathcal{C}\left[\frac{P_{1} \mathrm{a}_{11}^{2}}{\mathcal{N}_{o}}\right]-\mathcal{C}\left[\frac{P_{1} \mathrm{a}_{11}^{2}}{\eta+\mathcal{N}_{o}}\right]<\epsilon
$$

where $\epsilon$ is a carefully chosen threshold that dominates the threshold $\eta$. On the other hand, the secondary receiver gets the information as below

$$
\mathrm{y}_{2}=\mathrm{a}_{12} \mathrm{x}_{1}+\mathrm{a}_{22} \mathrm{x}_{2}+\mathrm{v}_{2}
$$

where $a_{12}, a_{22}$ stands for the channel gain between the secondary receiver and the transmitters, respectively. Then, what is the maximum achievable rate of $x_{2}$ in various wireless situations? What is the optimum setup of $\epsilon$ ? These questions need a satisfactory answer in the area of information theory.

\section{CAPacity Theorems with Channel Side INFORMATION}

This section aims to investigate Shannon capacity of the secondary link with respect to two aspects. One aspect is about the situation described by equations (2) and (4), while the other is about the situation, where the interference is completely removable, i.e., interference-free equivalent scenario.

\section{A. Achievable rate for a given threshold}

Eqn. (5) formulates a multiple-access environment, where $\mathrm{x}_{2}$ is the wanted information. If the secondary receiver deals with $x_{1}$ as noise, the achievable rate of $x_{2}$ is limited by

$$
R_{2}=I\left(\mathrm{x}_{2} ; \mathrm{y}_{2}\right)<\mathcal{C}\left[\frac{P_{2} \mathrm{a}_{22}^{2}}{P_{1} \mathrm{a}_{12}^{2}+\mathcal{N}_{o}}\right]
$$

where $I(;)$ denotes the mutual information. If the secondary receiver deals with $\mathrm{x}_{2}$ as message, the capacity region of this multiple access channel is given by [6]

$$
\bigcup\left(\begin{array}{l}
R_{1}<\mathcal{C}\left[\frac{P_{1} \mathrm{a}_{11}^{2}}{\eta+\mathcal{N}_{o}}\right], \\
R_{2}=I\left(\mathrm{x}_{2} ; \mathrm{y}_{2} \mid \mathrm{x}_{1}\right)<\mathcal{C}\left[\frac{P_{2} a_{22}^{2}}{\mathcal{N}_{o}}\right], \\
R_{1}+R_{2}=I\left(\mathrm{x}_{1}, \mathrm{x}_{2} ; \mathrm{y}_{2}\right)<\mathcal{C}\left[\frac{P_{1} \mathrm{a}_{12}^{2}+P_{2} \mathrm{a}_{22}^{2}}{\mathcal{N}_{o}}\right]
\end{array}\right) .
$$

Since the secondary communication does not influence the maximum achievable rate of the primary communication, the capacity region (7) indicates that the communication rate $R_{2}$ fulfills the following result

$$
\begin{aligned}
R_{2}<R_{2}^{(\mathrm{MAC})} \triangleq & \min \left(\mathcal{C}\left[\frac{P_{2} a_{22}^{2}}{\mathcal{N}_{o}}\right],\right. \\
& \left.\mathcal{C}\left[\frac{P_{1} \mathrm{a}_{12}^{2}+P_{2} \mathrm{a}_{22}^{2}}{\mathcal{N}_{o}}\right]-\mathcal{C}\left[\frac{P_{1} \mathrm{a}_{11}^{2}}{\eta+\mathcal{N}_{o}}\right]\right) .
\end{aligned}
$$

As a summary of (6) and (8), the achievable rate for a given threshold $\eta$ is

$$
R_{2}<\max \left(\mathcal{C}\left[\frac{P_{2} \mathrm{a}_{22}^{2}}{P_{1} \mathrm{a}_{12}^{2}+\mathcal{N}_{o}}\right], R_{2}^{(\mathrm{MAC})}\right) .
$$

Further calculation of (9) leads to the following result:

Theorem. 1: Given the channel condition $\mathrm{a}_{12}<\mathrm{a}_{11}$, the maximum achievable rate of the secondary link is given by (6). Otherwise, the maximum achievable rate is

$$
R_{2}<\mathcal{C}\left[\frac{P_{1} \mathrm{a}_{12}^{2}+P_{2} \mathrm{a}_{22}^{2}}{\mathcal{N}_{o}}\right]-\mathcal{C}\left[\frac{P_{1} \mathrm{a}_{11}^{2}}{\eta+\mathcal{N}_{o}}\right]
$$

for the channel condition $\mathrm{a}_{11}^{2}<\mathrm{a}_{12}^{2}<\mathrm{a}_{11}^{2}+\left(P_{2} \mathrm{a}_{22}^{2}\right) / \mathcal{N}_{o}$, and

$$
R_{2}<\mathcal{C}\left[\frac{P_{2} \mathrm{a}_{22}^{2}}{\mathcal{N}_{o}}\right]
$$

for $\mathrm{a}_{12}^{2}>\mathrm{a}_{11}^{2}+\left(P_{2} \mathrm{a}_{22}^{2}\right) / \mathcal{N}_{o}$.

Proof: See Appendix A. 


\section{B. Achievable rate for interference-free equivalent scenario}

In fact, eqn. (3) formulates a multiple-access channel. The primary receiver can reconstruct $\mathrm{x}_{2}$ without causing rate penalty to $\mathrm{x}_{1}$ only when the following condition holds [7]

$$
R_{2}<\mathcal{C}\left[\frac{P_{2} \mathrm{a}_{21}^{2}}{P_{1} \mathrm{a}_{11}^{2}+\mathcal{N}_{o}}\right]<\mathcal{C}\left[\frac{\eta}{P_{1} \mathrm{a}_{11}^{2}+\mathcal{N}_{o}}\right]
$$

In addition to Theorem. 1, (12) gives another upper bound of $R_{2}$. Therefore, the overall capacity limit of the secondary link is formulated by

$$
R_{2}<\max \left(\mathcal{C}\left[\frac{P_{2} \mathrm{a}_{22}^{2}}{P_{1} \mathrm{a}_{12}^{2}+\mathcal{N}_{o}}\right], R_{2}^{(\mathrm{MAC})}, \mathcal{C}\left[\frac{\eta}{P_{1} \mathrm{a}_{11}^{2}+\mathcal{N}_{o}}\right]\right) .
$$

The original proposal of IACR (4) indicates that $\eta$ is so small that the upper bound (13) reduces to (9).

\section{Optimum power control at the secondary transmitter}

The proposed power allocation is optimized for the capacity results provided in Theorem 1 . The first condition to configure $P_{2}$ is to meet the capacity difference $\epsilon$. This condition can be obtained by plugging $\eta=P_{2} \mathrm{a}_{21}^{2}$ into (4), i.e.,

$$
P_{2}<\frac{\left(4^{\epsilon}-1\right)\left(P_{1} \mathrm{a}_{11}^{2} / \mathcal{N}_{o}+1\right) \mathcal{N}_{o}}{\left(P_{1} \mathrm{a}_{11}^{2} / \mathcal{N}_{o}+1-4^{\epsilon}\right) \mathrm{a}_{21}^{2}} .
$$

Joint consideration of this result with Theorem. 1 leads to the following result:

Theorem. 2: Given the channel condition $\left(\mathrm{a}_{12}^{2}\right)>\left(\mathrm{a}_{11}^{2}+\right.$ $\left.\left(P_{2} \mathrm{a}_{22}^{2}\right) / \mathcal{N}_{o}\right)$, the transmit-power at the secondary transmitter is upper bounded by

$$
P_{2}<\min \left(\frac{\left(4^{\epsilon}-1\right)\left(P_{1} \mathrm{a}_{11}^{2} / \mathcal{N}_{o}+1\right) \mathcal{N}_{o}}{\left(P_{1} \mathrm{a}_{11}^{2} / \mathcal{N}_{o}+1-4^{\epsilon}\right) \mathrm{a}_{21}^{2}}, \frac{\left(\mathrm{a}_{12}^{2}-\mathrm{a}_{11}^{2}\right) \mathcal{N}_{o}}{\mathrm{a}_{12}^{2}}\right) .
$$

Otherwise, (14) is the upper bound of $P_{2}$.

$$
\text { Proof: See Appendix B. }
$$

\section{Localization Assisted Coexistence Strategies}

Section III shows that the IACR technique requires the channel side information. In particularly, the secondary transmitter needs the channel knowledge $a_{21}$, which is the key to determine whether to reuse the frequency band and how much power to spend. Basically, there are two channel estimation strategies that can be utilized to obtain this parameter. For the first strategy, the primary receiver performs estimation of $\mathrm{a}_{21}$ and feeds the parameter back to the secondary transmitter. In this case, the secondary transmitter needs to send data to the primary receiver for the purpose of channel estimation. However, the rate achievability of the primary link is considerably influenced due to the feedback overhead and the channel estimation process. For the second strategy, the secondary transmitter is responsible for the channel estimation, and the primary receiver temporarily becomes a transmitter. This is suitable for the situation, where the primary link operates in the time-division duplexing (TDD) fashion. However, the secondary link has to wait for the reverse communication of the primary link, which results in significant processing delay and overall network inefficiency. Therefore, to bypass the estimation of $a_{21}$ motivates us to utilize the localization information for the IACR.

In fact, exploiting localization information for cognitive radios is recently proposed in the article [8], which delivers a brainstorm about the potential help from positioning systems such as GPS and GNSS to the SSCR. The primary objective of this section is to investigate the localization assisted IACR network from the information-theoretic point-of-view. The investigation is based on the following basic assumptions:

A1) A map is available at the secondary transmitter;

A2) The positioning system informs the secondary transmitter regarding the accurate positions of other relevant network nodes;

A3) The secondary transmitter knows the p.d.f. of the channel gain $a_{21}$.

The coexistence strategy is described as follows:

The secondary transmitter performs frequency reuse only when it observes a large shadowing object between the primary receiver and itself (as a typical example shown in Fig. 1).

In this situation, non-line-of-sight (NLOS) propagation environment is assumed for the link between the secondary transmitter and the primary receiver, and thus the p.d.f. of $\mathrm{a}_{21}$ is assumed Rayleigh [9].

\section{A. Outage behavior of the primary communication}

Define $\bar{\gamma}_{21} \triangleq\left(P_{2} \sigma_{21}^{2}\right) /\left(\mathcal{N}_{o}\right)$ to be the average signal-tonoise ratio (SNR). $\sigma_{21}^{2}$ is the channel variance of the secondary transmitter to the primary receiver link, which can be obtained from the pre-established library of localization-dependent information [8]. The secondary communication occurs for the condition

$$
\bar{\gamma}_{21}<\bar{\gamma}_{\mathrm{t}}
$$

where $\bar{\gamma}_{21}$ denotes threshold of the average SNR. Hence, an appropriate setup of $\bar{\gamma}_{t}$ is important to the overall system performance.

The inequality (14) shows that the instantaneous SNR $\gamma_{21}$ is smaller than the threshold

$$
\gamma_{\mathrm{t}}=\frac{\left(4^{\epsilon}-1\right)\left(\gamma_{11}+1\right)}{\gamma_{11}+1-4^{\epsilon}}
$$

where $\gamma_{11}=\left(P_{1} \mathrm{a}_{11}^{2}\right) /\left(\mathcal{N}_{o}\right)$. Provided that the SNR $\gamma_{11}$ is deterministically known, the probability of the event $\left(\gamma_{21}>\right.$ $\left.\gamma_{t}\right)$ is given by [10]

$$
\operatorname{Pr}\left(\gamma_{21}>\gamma_{\mathrm{t}}\right)=\exp \left(-\frac{\gamma_{\mathrm{t}}}{\bar{\gamma}_{21}}\right)<\exp \left(-\frac{\gamma_{\mathrm{t}}}{\bar{\gamma}_{\mathrm{t}}}\right) .
$$

If the primary communication requires $\operatorname{Pr}\left(\gamma_{21}>\gamma_{\mathrm{t}}\right)<p_{\mathrm{t}}$, the SNR threshold $\bar{\gamma}_{\mathrm{t}}$ is obtained as below

$$
\bar{\gamma}_{\mathrm{t}}=\frac{\left(1-4^{\epsilon}\right)\left(\gamma_{11}+1\right)}{\left(\gamma_{11}+1-4^{\epsilon}\right) \ln \left(p_{\mathrm{t}}\right)} .
$$

This equation also gives an upper bound of the transmit-power at the secondary transmitter. 


\section{B. Outage behavior of the secondary communication}

Theorem. 2 shows that (14) is the upper bound of $P_{2}$ for the channel condition $\left(\mathrm{a}_{12}^{2}\right)<\left(\mathrm{a}_{11}^{2}+\left(P_{2} \mathrm{a}_{22}^{2}\right) / \mathcal{N}_{o}\right)$. In this situation, we can compare (19) with (14) and find that the capacity outage of the secondary link does not occur only if $\ln \left((1) /\left(p_{\mathrm{t}}\right)\right)>1$. Hence, the outage probability of the primary communication is upper bounded by

$$
p_{\mathrm{t}}<\exp (-1)
$$

Applying this outage probability in (18) results in $\bar{\gamma}_{\mathrm{t}}>\gamma_{\mathrm{t}}$.

For the channel condition $\left(\mathrm{a}_{12}^{2}\right)>\left(\mathrm{a}_{11}^{2}+\left(P_{2} \mathrm{a}_{22}^{2}\right) / \mathcal{N}_{o}\right)$, the transmit-power $P_{2}$ is upper bounded by (15). In this situation, $p_{\mathrm{t}}$ needs to be further reduced if the upper bound (15) is not the same as (14). Then, the above presentation can be summarized as follows:

Theorem. 3: Suppose the parameter $\bar{\gamma}_{21}$ is available, a necessary condition for the secondary communication to occur is $\bar{\gamma}_{21}<\gamma_{\mathrm{t}}$.

Remark. 1: Although Theorem. 3 only gives a necessary condition, a more strong condition can be obtained by considering $p_{\mathrm{t}}$ for the specific system requirements.

Remark. 2: If the secondary link does not occur for the condition $\mathrm{a}_{12}>\mathrm{a}_{11}$, then the secondary link will not have capacity outage for the condition $\bar{\gamma}_{21}<\gamma_{\mathrm{t}}$.

Remark. 3: Below provides the procedure about how to establish the secondary link with the support of localization information:

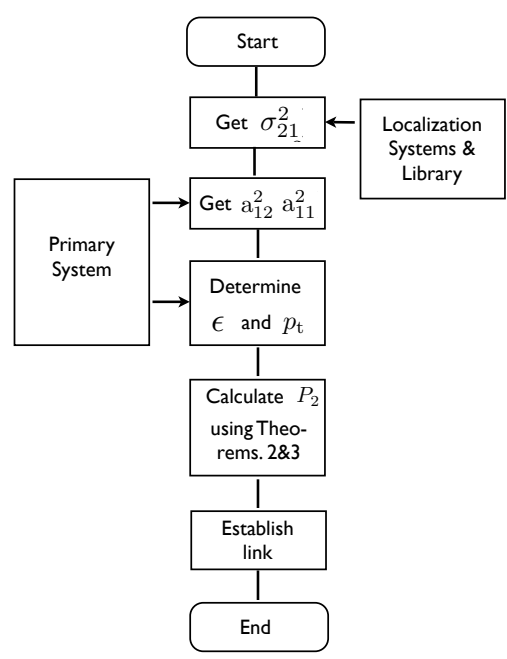

Fig. 2: The procedure of establishing the secondary link.

\section{NumERICAL RESUlts AND ANALYSIS}

The primary objectives of numerical analysis are in two folds: 1) to see the fundamental capacity limit of the IACR; 2) to see whether the localization information can offer satisfied performance for the IACR. The following channel parameters are given for the case study: $\mathrm{a}_{11}=1, \mathrm{a}_{22}=1, \mathrm{a}_{21}=0.1$, $0<\mathrm{a}_{12}<10$. As shown in (14), the transmit-power $P_{2}$

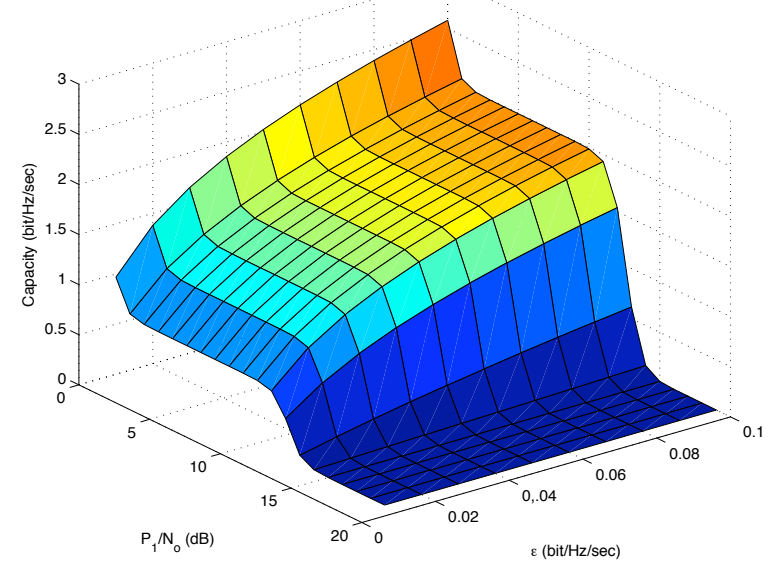

Fig. 3: Capacity of the secondary link as a function of $P_{1} / \mathcal{N}_{o}$ and $\epsilon$.

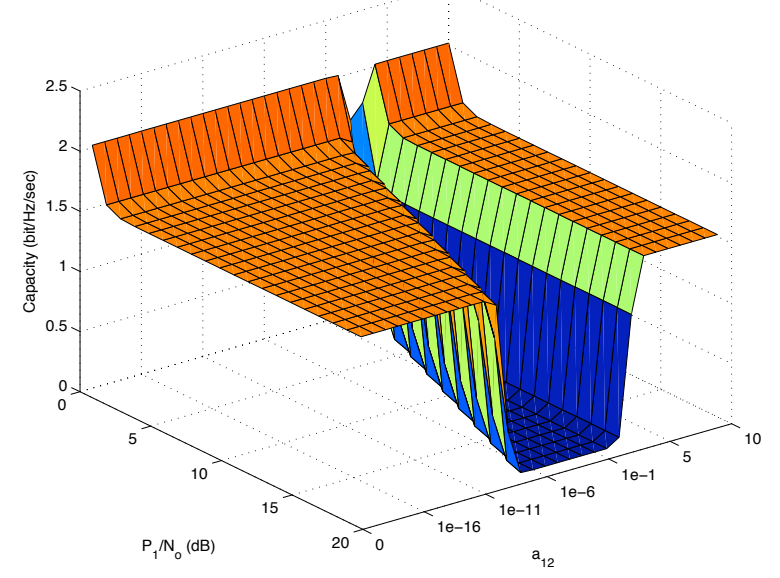

Fig. 4: Capacity of the secondary link as a function of $P_{1} / \mathcal{N}_{o}$ and $\mathrm{a}_{12}$.

is determined by SNR of the primary link $P_{1} / \mathcal{N}_{o}$. Hence, throughout the numerical analysis, the capacity results are always linked with the parameter $P_{1} / \mathcal{N}_{o}$.

Test Case 1: The objective of this test case is to see the relationship between the capacity of the secondary link and the rate penalty to the primary link $\epsilon$. In this test, the channel gain $\mathrm{a}_{12}$ is fixed to $1 \mathrm{e}-6$, and the rate penalty $\epsilon \in(0,0.1)$ bit/Hz/sec. The result plotted in Fig. 3 shows two phenomena: 1) the capacity of the secondary link generally decreases with increase of $P_{1} / N_{o}$, and drops to null for the range ( $>15$ $\mathrm{dB})$; 2) the capacity generally increases with increase of $\epsilon$ for the SNR range $(<15 \mathrm{~dB})$. These two phenomena reflects the resource competition behavior in coexistence environments.

Test Case 2: The objective of this test case is to see the relationship between the capacity of the secondary link and 

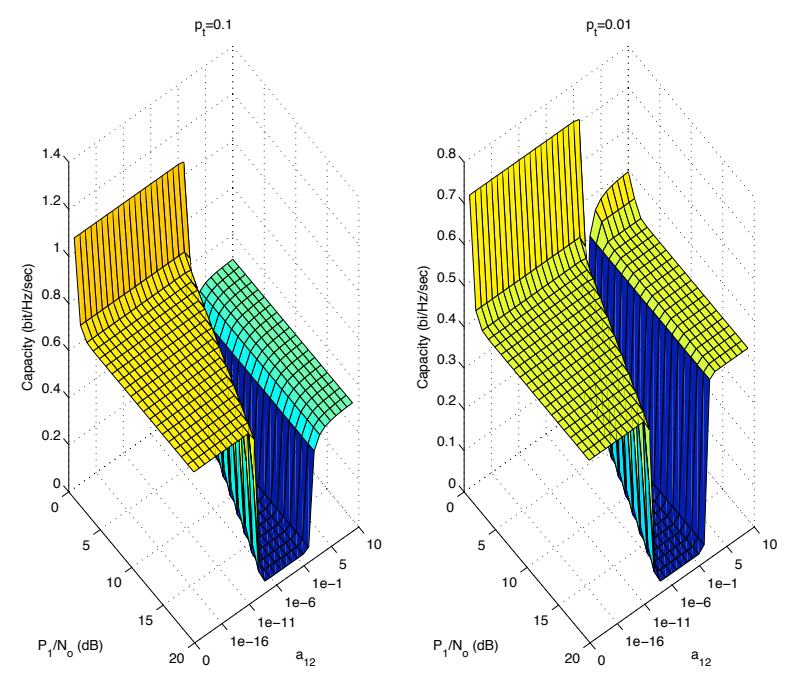

Fig. 5: Capacity of the secondary link with the localization information.

the channel gain $\mathrm{a}_{12}$. In this test, the rate penalty is fixed to $\epsilon=0.05$, and the channel gain $\mathrm{a}_{12} \in(0,10)$. Fig. 4 shows that the capacity keeps almost constant for the SNR range $(>2 \mathrm{~dB})$. However, a capacity gap is observed for the range $\mathrm{a}_{12} \in(1 \mathrm{e}-7,2)$. This gap becomes small with the decrease of $P_{1}$. This phenomenon is due to the nature of interference, i.e., both very large and very small interference show the identical impact on the system performance [11].

Test Case 3: The objective of this test case is to see the capacity limit without the knowledge of $\mathrm{a}_{21}$ but with the knowledge of $\sigma_{21}^{2}$, which is set to $\sigma_{21}^{2}=0.02$. Fig. 5 shows the capacity results for two cases, i.e., $p_{\mathrm{t}}=0.1$ and $p_{\mathrm{t}}=0.01$. In contrast with Fig. 4, the localization-assisted IACR shows more than $1 \mathrm{bit} / \mathrm{Hz} / \mathrm{sec}$ loss in rate $\left(\right.$ see $\left.p_{\mathrm{t}}=0.1\right)$. Further reducing $p_{\mathrm{t}}$ to 0.01 leads to more loss in transmission rate (more than $0.2 \mathrm{bit} / \mathrm{Hz} / \mathrm{sec}$ ).

\section{CONCLUSION}

In this paper, we have investigated capacity theorems for the IACR system in two scenarios. One scenario was about the situation, where the secondary transmitter had the perfect channel side information. The other was about the situation, where the secondary transmitter did not know the channel between the primary receiver and itself, but obtained the channel variance from localization systems. Numerical results have shown that the localization information could help the IACR system, but could not offer comparable performance with the case with perfect channel side information.

\section{APPENDIX A: PROOF OF THEOREM. 1}

Due to the fact

$$
\mathcal{C}\left[\frac{P_{2} a_{22}^{2}}{\mathcal{N}_{o}}\right]>\mathcal{C}\left[\frac{P_{2} \mathrm{a}_{22}^{2}}{P_{1} \mathrm{a}_{12}^{2}+\mathcal{N}_{o}}\right]
$$

(6) is not the upper bound only when the following two conditions hold

$$
\begin{gathered}
\mathcal{C}\left[\frac{P_{2} \mathrm{a}_{22}^{2}}{P_{1} \mathrm{a}_{12}^{2}+\mathcal{N}_{o}}\right]<\mathcal{C}\left[\frac{P_{1} \mathrm{a}_{12}^{2}+P_{2} \mathrm{a}_{22}^{2}}{\mathcal{N}_{o}}\right]-\mathcal{C}\left[\frac{P_{1} \mathrm{a}_{11}^{2}}{\eta+\mathcal{N}_{o}}\right] \\
\mathcal{C}\left[\frac{P_{2} a_{22}^{2}}{\mathcal{N}_{o}}\right]<\mathcal{C}\left[\frac{P_{1} \mathrm{a}_{12}^{2}+P_{2} \mathrm{a}_{22}^{2}}{\mathcal{N}_{o}}\right]-\mathcal{C}\left[\frac{P_{1} \mathrm{a}_{11}^{2}}{\eta+\mathcal{N}_{o}}\right]
\end{gathered}
$$

The inequality (22) leads to the condition $\mathrm{a}_{12}>\mathrm{a}_{11}$, and (23) leads to the condition $\mathrm{a}_{12}^{2}>\mathrm{a}_{11}^{2}+\left(P_{2} \mathrm{a}_{22}^{2}\right) / \mathcal{N}_{o}$. Based on these results, Theorem. 1 can be straightforwardly obtained.

\section{APPENDIX B: PROOF OF THEOREM. 2}

For the channel condition $\mathrm{a}_{12}<\mathrm{a}_{11}$, the maximum achievable rate is given by (6). In this case, (14) is the optimum power setup. If the upper bound of capacity is (10), Theorem. 1 shows that the following condition must hold

$$
\frac{\left(\mathrm{a}_{12}^{2}-\mathrm{a}_{11}^{2}\right) \mathcal{N}_{o}}{\mathrm{a}_{22}^{2}}<P_{2}<\frac{\left(4^{\epsilon}-1\right)\left(P_{1} \mathrm{a}_{11}^{2} / \mathcal{N}_{o}+1\right) \mathcal{N}_{o}}{\left(P_{1} \mathrm{a}_{11}^{2} / \mathcal{N}_{o}+1-4^{\epsilon}\right) \mathrm{a}_{21}^{2}} .
$$

If the upper bound of capacity is (11), Theorem. 1 indicates that $P_{2}$ is upper bounded by

$$
P_{2}<\frac{\left(\mathrm{a}_{12}^{2}-\mathrm{a}_{11}^{2}\right) \mathcal{N}_{0}}{\mathrm{a}_{22}^{2}} .
$$

In this case, the optimum transmite-power is (15).

\section{ACKNOWLEDGMENT}

This work has been performed in the framework of European Commission ICT-WHERE project, which was supported in part by the European Union. Na Yi was supported in part by the UK MVCE Core 4 under Efficiency programme, which was funded in part by EPSRC.

\section{REFERENCES}

[1] National Telecommunications and Information Administration, "FCC frequency allocation chart", Download available at www.ntia.doc.gov/osmhome/allochrt.pdf., 2003.

[2] R. Tafazolli (Editor), Technologies for the wireless future (volumn 2), Wiley, England, 2006.

[3] J. Mitola III and G. Q. Maguire Jr., "Cognitive radio: making software radio more personal”, IEEE Personal Communs., pp. 13-18, August 1999.

[4] FCC Spectrum Policy Task Force, "Report of the spectrum efficiency working group", Technical report 02-135, Nov. 2002.

[5] I. Maric, R. D. Yates, and G. Kramer, "Capacity of interference channels with partial transmitter cooperation", IEEE Trans. Inf. Theory, vol. 53, pp. 3526-3548, Oct. 2007.

[6] T. M. Cover and J. A. Thomas, Element of Information Theory, Wiley, New York, second edition, 2006.

[7] A. B. Carleial, "A case where interference does not reduce capacity", IEEE Trans. Inf. Theory, vol. IT-21, pp. 569-570, Sept. 1975.

[8] S. Yarkan and H. Arslan, "Exploiting location awareness toward improved wireless system design in cognitive radio", IEEE Commun. Mag., vol. 53, pp. 128-136, Jan. 2008.

[9] T. S. Rappaport, Wireless communications: principles and practice, Prentice Hall PTR, New Jersey, USA, 1996.

[10] M. K. Simon and M-S. Alouini, Digital communication over fading channel, Wiley \& Son, Canada, 2nd edition, 2005.

[11] H. Sato, "The capacity of gaussian interferene channel under strong interference", IEEE Trans. Inf. Theory, vol. IT-27, pp. 786-788, Nov. 1981. 